\title{
Gravidez Gemelar com uma Mola Hidatiforme Completa e um Co-Gémeo Viável
}

\author{
Twin Pregnancy with a Complete Hydatiform Mole and a Viable Co-Twin
}

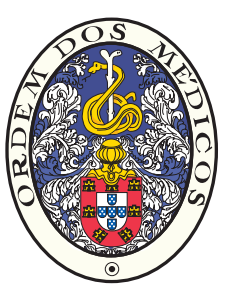

\author{
Ana Beatriz GODINHO ${ }^{1}$, Diana MARTINS ${ }^{1}$, Cláudia ARAÚJO ${ }^{1}$, Maria Antonieta MELO², Luís MENDES GRAÇA ${ }^{1}$ \\ Acta Med Port 2014 Jan-Feb;27(1):135-137
}

\section{RESUMO}

A existência de uma gravidez gemelar bicoriónica com uma mola hidatiforme completa e um co-gémeo viável é um evento raro, sendo o diagnóstico habitualmente realizado no segundo trimestre. Pode associar-se a tirotoxicose, pré-eclâmpsia, hemorragia vaginal, doença persistente do trofoblasto e morte fetal. Os autores descrevem o caso de uma grávida com hemorragia do primeiro trimestre. As imagens ecográficas foram interpretadas como uma gravidez gemelar bicoriónica com um embrião vivo e outra placenta com imagem de descolamento. Às 16 semanas visualizou-se um feto vivo com placenta posterior e uma massa vacuolada anterior, vascularizada. Perante a hipótese de doença do trofoblasto o casal optou pela interrupção da gravidez. O exame anatomo-patológico confirmou o diagnóstico. Perante a raridade desta entidade clínica, é necessário um elevado índice de suspeição para estabelecer o seu diagnóstico ecográfico. Apesar de estarem descritos casos com um bom desfecho materno e neonatal, a decisão de continuação da gravidez deve ser tomada pelos progenitores.

Palavras-chave: Doenças em Gémeos; Gravidez Gemelar; Mola Hidatiforme; Ultrassonografia.

\section{ABSTRACT}

A complete hydatiform mole coexisting with a live, viable twin is a rare event. The diagnosis is challenging, and is normally achieved only at second trimester. It may be associated with thyrotoxicosis, vaginal bleeding, preeclampsia, fetal death or persistent throphoblastic disease. The authors describe the case of a pregnant woman presenting with first trimester bleeding. Ultrasound revealed a twin pregnancy with a viable twin and another placenta apparently detached. At 16 gestational weeks ultrasound revealed a live fetus with a normal placenta and a separate vacuolated and vascularized mass. Facing the hypothesis of gestational trophoblastic disease, the couple chose pregnancy interruption. Given the rarity of this situation, a high index of suspicion is needed to achieve the diagnosis. Despite the existence of case reports with good fetal and maternal outcome, the decision of pregnancy continuation should be made by the informed parents.

Keywords: Diseases in Twins; Hydatidiform Mole; Pregnancy, Twin; Ultrasonography.

\section{INTRODUÇÃO}

A doença trofoblástica gestacional define-se como uma doença proliferativa das células do trofoblasto resultantes de um processo de fertilização aberrante. As doenças do trofoblasto mais frequentes são a mola hidatiforme completa, caracterizada por edema generalizado do tecido vilositário, hiperplasia trofoblástica difusa, sem tecido embrionário presente, e a mola hidatiforme parcial, caracterizada por alterações placentárias hidatiformes focais e presença de embrião, habitualmente triplóide, com múltiplas malformações. ${ }^{1}$ Estima-se que a incidência de mola hidatiforme seja de 1 para 1 000, embora se verifiquem diferenças geográficas importantes, com uma maior incidência nos países asiáticos e da América do Sul. ${ }^{1}$ Esta patologia é considerada pré-maligna, pelo que a interrupção da gravidez é a regra assim que se estabelece o diagnóstico.

A mola hidatiforme completa está associada a várias complicações maternas, como a tirotoxicose, hiperemese gravídica, quistos bilaterais dos ovários, pré-eclâmpsia precoce, eclâmpsia, hemorragia vaginal e doença persistente do trofoblasto..$^{2,3}$ Associa-se também a morte fetal. ${ }^{2,3}$

A existência de uma gravidez gemelar bicoriónica, em que coexiste uma mola hidatiforme completa com um gé- meo viável, é um evento raro, estimando-se a sua incidência em 1:20 000 a 1:100 $000 .^{2} \mathrm{O}$ primeiro caso descrito na literatura remonta a $1952,{ }^{4}$ e desde então têm sido publicados casos clínicos isolados ${ }^{5-7}$ e algumas séries de casos. $2,3,8$

Os autores apresentam o caso de uma gravidez gemelar bicoriónica com uma mola hidatiforme completa e um co-gémeo viável.

\section{CASO CLÍNICO}

Primípara saudável, de 31 anos, grupo sanguíneo $\mathrm{ARh}+$. Iniciou hemorragia vaginal escassa às nove semanas de gravidez, sem outros sintomas associados. As imagens ecográficas sugeriam gravidez gemelar bicoriónica com um embrião vivo e uma outra placenta com imagem sugestiva de descolamento (Fig. 1) mas sem saco amniótico nem embrião. Às 12 semanas mantinha hemorragia vaginal escassa, e a ecografia mostrava um feto vivo de morfologia normal, com os marcadores ecográficos de aneuploidias negativos e com placenta posterior, e uma imagem sugestiva de descolamento placentário junto ao orifício interno do colo (Fig. 2).

1. Departamento de Obstetrícia, Ginecologia e Medicina da Reprodução. Hospital de Santa Maria. Centro Hospitalar Lisboa Norte. Lisboa. Portugal.

2. Unidade de Ecografia. Departamento de Obstetrícia, Ginecologia e Medicina da Reprodução. Hospital de Santa Maria. Centro Hospitalar Lisboa Norte. Lisboa. Portugal. Recebido: 30 de Janeiro de 2013 - Aceite: 24 de Agosto de 2013 | Copyright @ Ordem dos Médicos 2014 


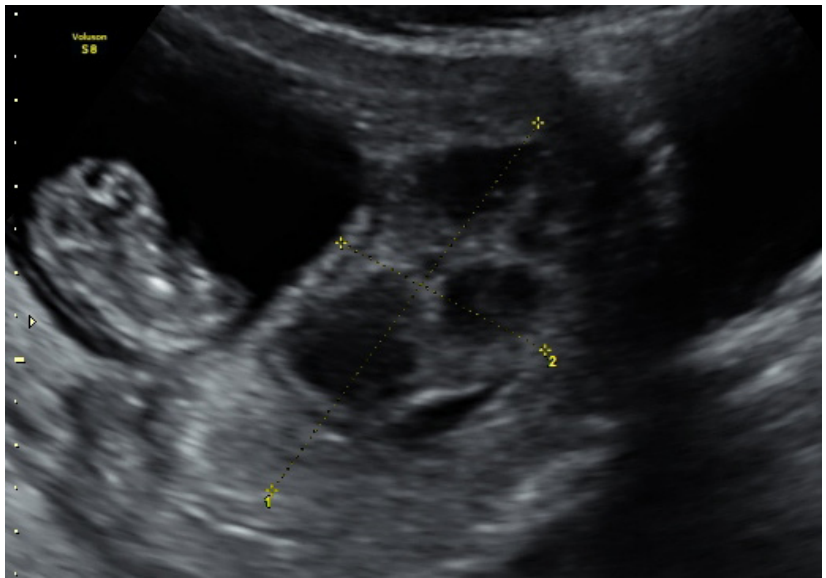

Figura 1 - Imagem ecográfica às 9 semanas de gestação, sugestiva de gravidez gemelar bicoriónica com um embrião vivo e um saco gestacional sem embrião, com descolamento

Às 16 semanas, uma semana após a suspensão das perdas hemáticas, a ecografia revelou um feto vivo, com biometria adequada, boa dinâmica, placenta posterior, e uma massa vacuolada, vascularizada, inserida na face anterior do útero (Fig. 3). Perante a hipótese diagnóstica de uma gravidez gemelar com uma mola hidatiforme com um co-gémeo viável, o casal foi esclarecido acerca das possíveis complicações maternas e fetais e da sua gravidade, tendo solicitado a interrupção médica da gravidez.

A interrupção foi realizada às 17 semanas com misoprostol por via vaginal e decorreu sem complicações. Verificou-se a expulsão de um feto sem malformações aparentes, uma placenta normal e uma massa vesiculosa macroscopicamente compatível com mola hidatiforme (Fig. 4). Procedeu-se a aspiração uterina sob controlo ecográfico, para assegurar a completa expulsão do produto de concepção. A doente teve alta no dia seguinte, clinicamente bem.

A doente manteve-se sempre assintomática, com valores tensionais normais. A radiografia de tórax e a função tiroideia mantiveram-se sem alterações. Os valores de HCG normalizaram ao fim de seis semanas após a interrupção.

Foi realizado o exame anatomo-patológico do produto de concepção, que confirmou o diagnóstico.

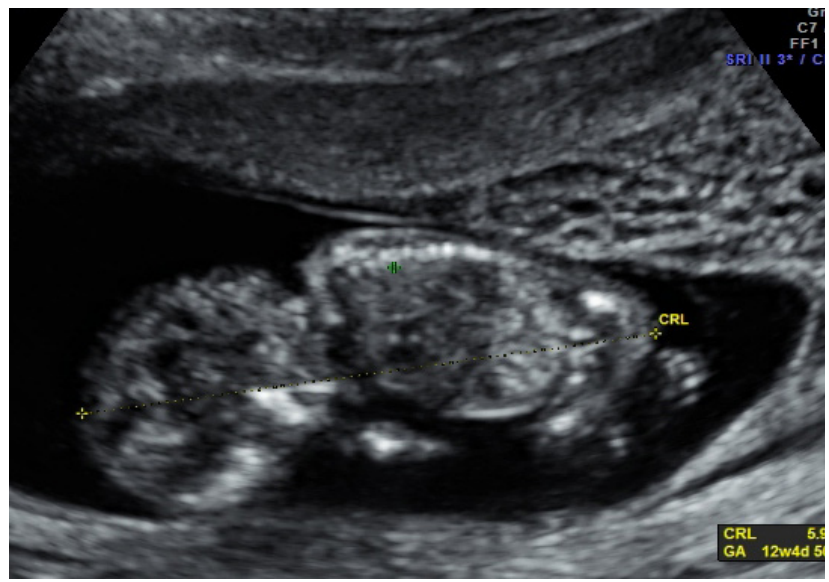

Figura 2 - Imagem ecográfica às 12 semanas, com um feto vivo e uma imagem sugestiva de descolamento placentário

\section{DISCUSSÃO}

A coexistência de uma mola hidatiforme completa com um feto viável coloca várias questões quanto ao diagnóstico, terapêutica e prognóstico.

A maior série foi publicada por Sebire ${ }^{2}$ em 2002, tendo sido analisados 77 casos. Os autores concluíram que estas gestações representam um maior risco de desenvolvimento de pré-eclâmpsia, tirotoxicose, hemorragia vaginal, tromboembolismo pulmonar e doença trofoblástica gestacional persistente. Por outro lado, nas grávidas que decidiram continuar a gestação, em apenas $40 \%$ o recém-nascido foi viável, dada a elevada taxa de morte fetal e de necessidade de interrupção da gravidez pré-viável por complicações maternas graves. Neste estudo não houve incidência significativamente aumentada de doença trofoblástica gestacional persistente em comparação com os casos de mola hidatiforme completa isolada (16\%) contrariando os achados publicados em 2000 por Matsui. ${ }^{3}$ Neste estudo foram investigados 72 casos de gravidez gemelar com uma mola hidatiforme completa e um gémeo viável, tendo concluído que 30 a $50 \%$ dos casos evoluem para doença trofoblástica gestacional persistente, por oposição aos $12,5 \%$ nos casos de mola hidatiforme completa isolada.

Perante a hipótese de diagnóstico de uma gravidez

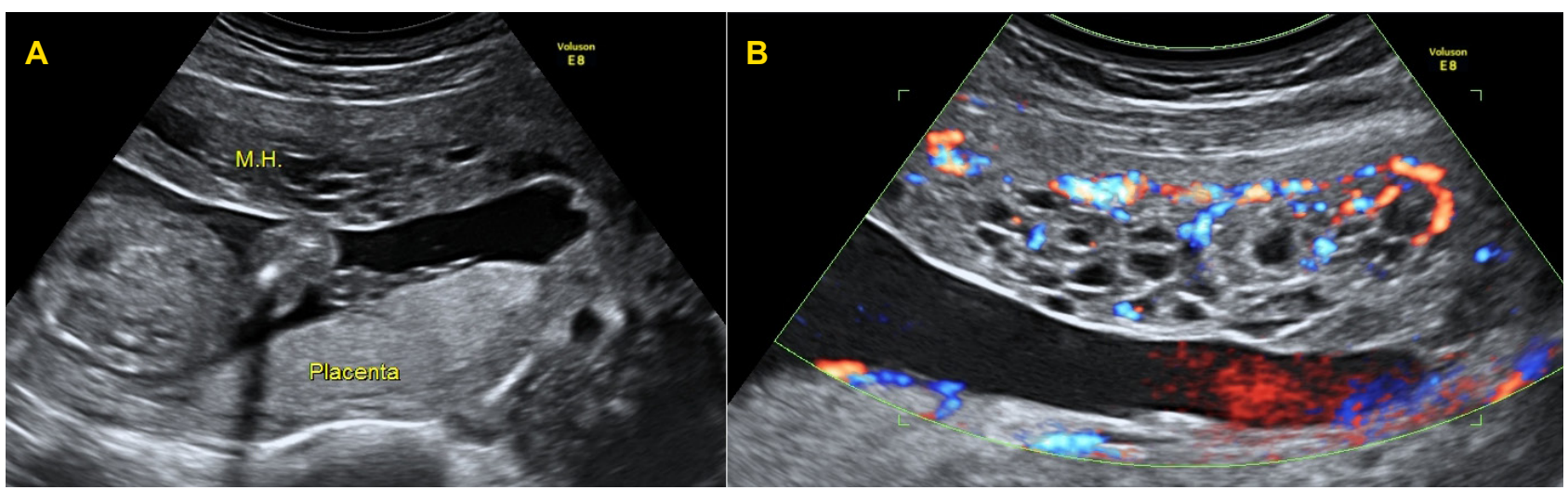

Figura 3A - Imagem ecográfica às 16 semanas, com um feto vivo, uma placenta normal e uma placenta molar Figura 3B - Vascularização da placenta molar 


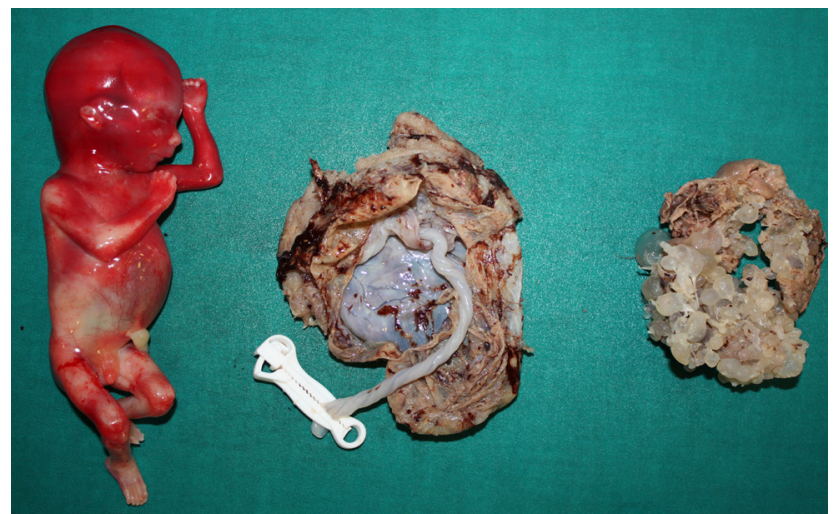

Figura 4 - Produto de concepção: feto, placenta e mola hidatiforme (fotografia cortesia de Artur Costa e Silva)

gemelar em que há uma mola hidatiforme completa e um feto viável, o casal tem de optar entre interromper a gravidez de um feto vivo, sem patologia, ou deixar prosseguir a gestação, enfrentando os riscos de morte fetal e de complicações maternas graves. Se o casal optar por prosse-

\section{REFERÊNCIAS}

1. Altieri A, Franceschi S, Ferlay J, Smith J, La Vecchia C. Epidemiology and aetiology of gestational throphoblastic diseases. Lancet Oncol. 2003;4:670-8

2. Sebire NJ, Foskett M, Paradinas FJ, Fisher RA, Francis RJ, Short D, et al. Outcome of twin pregnancies with complete hydatiform mole and healthy co-twin. Lancet. 2002;359:2165-6.

3. Matsui H, Sekiya S, Hando T, Wake N, Tomoda Y. Hydatiform mole coexistent with a twin live fetus: a national collaborative study in Japan. Hum Reprod. 2000;15:608-11.

4. Taylor JH. Twin pregnancy: Well-formed hydatiform mole associated with normal viable child. BMJ. 1957;1:1103.

5. Piura B, Rabinovich A, Hershkovitz R, Maor E, Mazor M. Twin pregnancy with a complete hydatiform mole and a surviving co-existent fetus. Arch Gynecol Obstet. 2008;278:377-82. guir a gravidez, a grávida deve ser referenciada para um centro perinatal diferenciado, e as complicações maternas devem ser rastreadas, com a vigilância da função tiroideia, hemorragia vaginal, sinais e sintomas sugestivos de pré-eclâmpsia e parto pré-termo. Alguns autores ${ }^{9}$ sugerem a realização de radiografia torácica trimestral para pesquisar metastização pulmonar. O casal deve ser informado que as hipóteses de ter um parto de um recém-nascido vivo e saudável são inferiores a $50 \%$, e que entre 16 a $50 \%$ dos casos desenvolvem doença trofoblástica gestacional persistente..$^{2,3}$

\section{CONFLITOS DE INTERESSE}

Os autores declaram a inexistência de conflitos de interesse na realização do presente trabalho.

\section{FONTES DE FINANCIAMENTO}

Não existiram fontes externas de financiamento para a realização deste artigo.

6. Marcorelles P, Audrezet MP, Le Bris MJ, Laurent Y, Chabaud JJ, Ferec $\mathrm{C}$, et al. Diagnosis and outcome of complete hydatiform mole coexisting with a live twin fetus. Eur J Obstet Gynecol Reprod Biol 2005;118:21-7.

7. Malhotra N, Deka D, Takkar D, Kochar S, Goel S, Sharma MC. Hydatiform mole with a coexisting live fetus in dichorionic twin gestation. Eur J Obstet Gynecol Reprod Biol. 2001;94:301-3.

8. Choi-Hong SR, Genest DR, Crum CP, Berkowitz E, Goldstein DP, Schofield $\mathrm{DH}$. Twin pregnancies with complete hydatiform mole and coexisting fetus: Use of fluorescent in situ hybridization to evaluate placental X- and Y- cromossomal content. Hum Pathol. 1995;26:1175-80.

9. Wee $L$, Jauniaux $E$. Prenatal diagnosis and management of twin pregnancies complicated by a co-existing molar pregnancy. Prenat Diagn. 2005;25:772-6 


\section{Gravidez Gemelar com uma Mola Hidatiforme Completa e um Co-Gémeo Viável}

Acta Med Port 2014:27:135-137

Publicado pela Acta Médica Portuguesa, a Revista Científica da Ordem dos Médicos

Av. Almirante Gago Coutinho, 151

1749-084 Lisboa, Portugal.

Tel: +351218428215

E-mail: submissao@actamedicaportuguesa.com

www.actamedicaportuguesa.com

ISSN:0870-399X | e-ISSN: 1646-0758

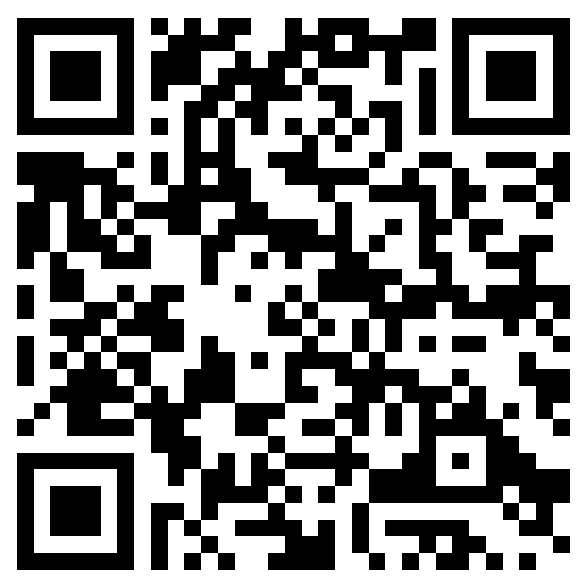

